\title{
Hybrid iterative wavefront shaping for high-speed focusing through scattering media
}

\author{
Ashton S. Hemphill, Lihong V. Wang
}

Ashton S. Hemphill, Lihong V. Wang, "Hybrid iterative wavefront shaping for high-speed focusing through scattering media," Proc. SPIE 9717, Adaptive Optics and Wavefront Control for Biological Systems II, 97170V (15 March 2016); doi: 10.1117/12.2211434

SPIE. Event: SPIE BiOS, 2016, San Francisco, California, United States 


\title{
Hybrid iterative wavefront shaping for high-speed focusing through scattering media
}

\author{
Ashton S. Hemphill, Lihong V. Wang ${ }^{*}$ \\ Washington University in St. Louis \\ Department of Biomedical Engineering, Optical Imaging Laboratory, Campus Box 1097, 1 \\ Brookings Drive, Saint Louis, Missouri 63130, USA
}

\begin{abstract}
A major limiting factor of optical imaging in biological applications is the diffusion of light by tissue, preventing focusing at depths greater than $\sim 1 \mathrm{~mm}$ in the body. To overcome this issue, phase-based wavefront shaping alters the phase of sections of the incident wavefront to counteract aberrations in phase caused by scattering. This enables focusing through scattering media beyond the optical diffusion limit and increases signal compared to amplitude-based compensation. However, in previous studies, speed of optimization has typically been limited by the use of a liquid crystal spatial light modulator (SLM) for measurement and display. SLMs usually have refresh rates of less than $100 \mathrm{~Hz}$ and require much longer than the speckle correlation time of tissue in vivo, usually on the order of milliseconds, to determine the optimal wavefront. Here, we present a phase-based iterative wavefront shaping method based on an onaxis digital micromirror device (DMD) in conjunction with an electro-optic modulator (EOM) for measurement and a fast SLM for display. By combining phase modulation from an EOM with the modal selection of the DMD, we take advantage of DMDs higher refresh rate, approximately $23 \mathrm{kHz}$, for iterative phase measurement. The slower SLM requires one update for display following the rapid determination of the optimal wavefront via the DMD, allowing for high-speed wavefront shaping. Using this system, we are able to focus through scattering media using 64 modes in under 8 milliseconds, on the order of the speckle correlation time for tissue in vivo.
\end{abstract}

Keywords: Iterative Wavefront Shaping, High-Speed Wavefront Shaping, Turbid Media, Active or Adaptive Optics

\section{INTRODUCTION}

Light plays a crucial role in high-resolution imaging ${ }^{1-3}$, as well as the accurate delivery of energy for applications such as optogenetics ${ }^{4}$, microsurgery ${ }^{5}$ and photothermal therapy ${ }^{6}$. However, photons experience scattering in biological tissue, preventing focusing using lenses at much beyond the optical diffusion limit, approximately $1 \mathrm{~mm}$ in vivo ${ }^{7}$. Because of this, the feasible depth of optical imaging and therapeutic techniques are greatly limited.

Several methods have recently been developed to overcome the optical diffusion limit through wavefront shaping (WFS), specifically iterative WFS and optical time reversal. Iterative WFS ${ }^{8-14}$ functions by measuring the transmission matrix of the scattering medium, or a subset thereof ${ }^{15}$, iteratively over the input modes of the incoming wavefront. Using this information, the phase is then modified using a liquid crystal on silicon (LCoS) spatial light modulator (SLM) to correct the heterogeneous aberrations caused by random scattering within the medium. By optimizing the phase, constructive interference is formed at the desired location, generating a focus. In optical time reversal, an internal guide star (either real ${ }^{16-18}$ or virtual ${ }^{19-21}$ ) is used to provide feedback for phase-conjugation. By conjugating the phase at the guide star through a method such as optical phase conjugation (OPC), the wavefront converges to its origin, forming a focus at the guide star through constructive interference.

However, living tissue is dynamic, causing the inhomogeneities that produce scattering to shift and decorrelate rapidly, on the order of milliseconds in vivo ${ }^{22,23}$. Thus, in order for the collected phase information to be accurate, it is ideal that wavefront optimization and display be completed in, at most, $10 \mathrm{~ms}^{24}$.

Digital optical phase conjugation, that is OPC utilizing an SLM, shows good enhancement ${ }^{16,20,21}$. However, it suffers

*1hwang@wustl.edu, 1-314-935-6152

Adaptive Optics and Wavefront Control for Biological Systems II, edited by Thomas G. Bifano, Joel Kubby, Sylvain Gigan, Proc. of SPIE Vol. 9717, 97170V · (C) 2016 SPIE · CCC code: 1605-7422/16/\$18 · doi: 10.1117/12.2211434 
from slow speed $(100 \mathrm{~ms})^{25}$ due to the low refresh rate of the LCOS SLM, typically less than $300 \mathrm{~Hz}$, as well as image acquisition time. Combined with the need for data transfer and processing, optimization speed is generally insufficient for focusing on the order of the speckle correlation time in living tissue. Conversely, analog OPC utilizing a photorefractive crystal ${ }^{19,26-28}$ provides extremely fast optimization; $10 \mathrm{~ms}$ for $10^{7}$ modes $^{27}$. However, this method produces an energy gain below unity, reducing its utility in many applications.

Iterative wavefront shaping likewise must compromise efficacy in order to achieve higher speeds. In phase-based wavefront shaping an SLM is typically utilized, resulting in very low speed as the SLM must be updated for each iteration. Binary amplitude-based wavefront shaping may choose to utilize a much faster digital micromirror device $(\mathrm{DMD})^{8,13,14}$, updating on the order of KHz. However, because DMDs may only turn an input mode on or off, the maximum enhancement provided is reduced by a factor of $5^{14,29}$, lowering its utility in biological applications that suffer from poor signal-to-noise ratio and hampering techniques which require higher energy. Finally, a DMD may be used in phase-based wavefront shaping by utilizing off-axis Lee holograms to generate the appropriate phase at each input mode. This allows for phase-based wavefront shaping at the speed of the $\mathrm{DMD}^{13}$, but necessitates sacrificing $\sim 80 \%$ of the illumination incident to the $\mathrm{DMD}^{9,29}$. This again results in lowered signal and precludes use of the method in a variety of applications.

Here we present a hybrid method of iterative wavefront shaping capable of phase-based optimization at the speed of the DMD without sacrificing illumination as required for off-axis holography. The DMD provides modal isolation at the surface of the SLM, sequentially illuminating each superpixel. The EOM is utilized to rotate the phase as each mode is isolated, allowing the optimal phase to be measured, calculated and displayed with a single update to the SLM. In doing so, we are able to complete the optimization, i.e. measure, calculate, display and record the corrected wavefront, in less than 8 ms using 64 input modes, on the order of the speckle correlation time in living tissue.

\section{METHODS}

\subsection{Principle}

The hybrid system functions by eliminating the need for the slower LCoS SLM in the iterative process of measuring the optimal phase at each input mode. Instead, the much faster DMD is used to provide modal isolation through binary amplitude modulation. As shown in Fig. 1, the surface of the DMD is imaged to that of the SLM, with each superpixel of the DMD corresponding to a superpixel at the SLM. Thus, during measurement each mode can be independently illuminated by the DMD while an EOM provides phase rotation of the reference arm. During this process the SLM is held flat and requires no updates. After all input modes have been measured by the DMD in conjunction with the EOM, the optimal wavefront is calculated and displayed by the SLM while the DMD is switched all on to fully illuminate the SLM. In this way, the measurement of the phase is done at the speed of the DMD, with the overall optimization process requiring only a single refresh by the SLM. Additionally, this method is purely on-axis, meaning that full illumination is retained and transferred to the sample without the need to sacrifice energy as with off-axis Lee holograms.

\subsection{Experimental Set-Up}

As shown in Fig. 1, illumination was provided by a $5 \mathrm{~W}$ continuous wave laser (Verdi V5, Coherent, USA) at $532 \mathrm{~nm}$. The beam was vertically polarized before the first non-polarizing beamsplitter split it into sample and reference arms. The beam in the sample arm was then expanded by the first pair of lenses to fill the DMD (DLP 7000, Texas Instruments, USA). Following the DMD, the beam was then imaged to the surface of the SLM (HSP256-0532, Meadowlark Optics, USA) and slightly reduced for pixel matching by a pair of lenses (LA1433-A, Thorlabs, USA \& KPX091AR.14, Newport, USA). The beam was then directed to the second beam splitter where it was recombined with the reference arm for interferometry. Meanwhile, after leaving the first beam splitter the reference arm was passed through a phase modulating EOM (350-105, Conoptics, USA). A $1.25 \mathrm{Vpp}$ ramp function was supplied at $62.5 \mathrm{KHz}$, three times per iteration, by a function generator (DS345, Stanford Research Systems, USA) and amplified by a 375 gain high voltage linear amplifier (M302RM, Conoptics, USA). The beam was then expanded by a pair of lenses to match the sample beam leaving the SLM and recombined at the second beam splitter. The combined beams were then focused by a lens (LA1951-A, Thorlabs, USA) onto the ground glass diffuser (DG10-220, Thorlabs, USA). After the diffuser, the beam was allowed to diverge before a single speckle grain was isolated via an adjustable iris (SM1D12, Thorlabs, USA) mounted on the chassis of the photodiode (PDA36A, Thorlabs, USA). The light was then detected by the photodiode and amplified by $40 \mathrm{~dB}$ before being digitized by a data acquisition (DAQ) card (ATS9462, AlazarTech, Canada). 


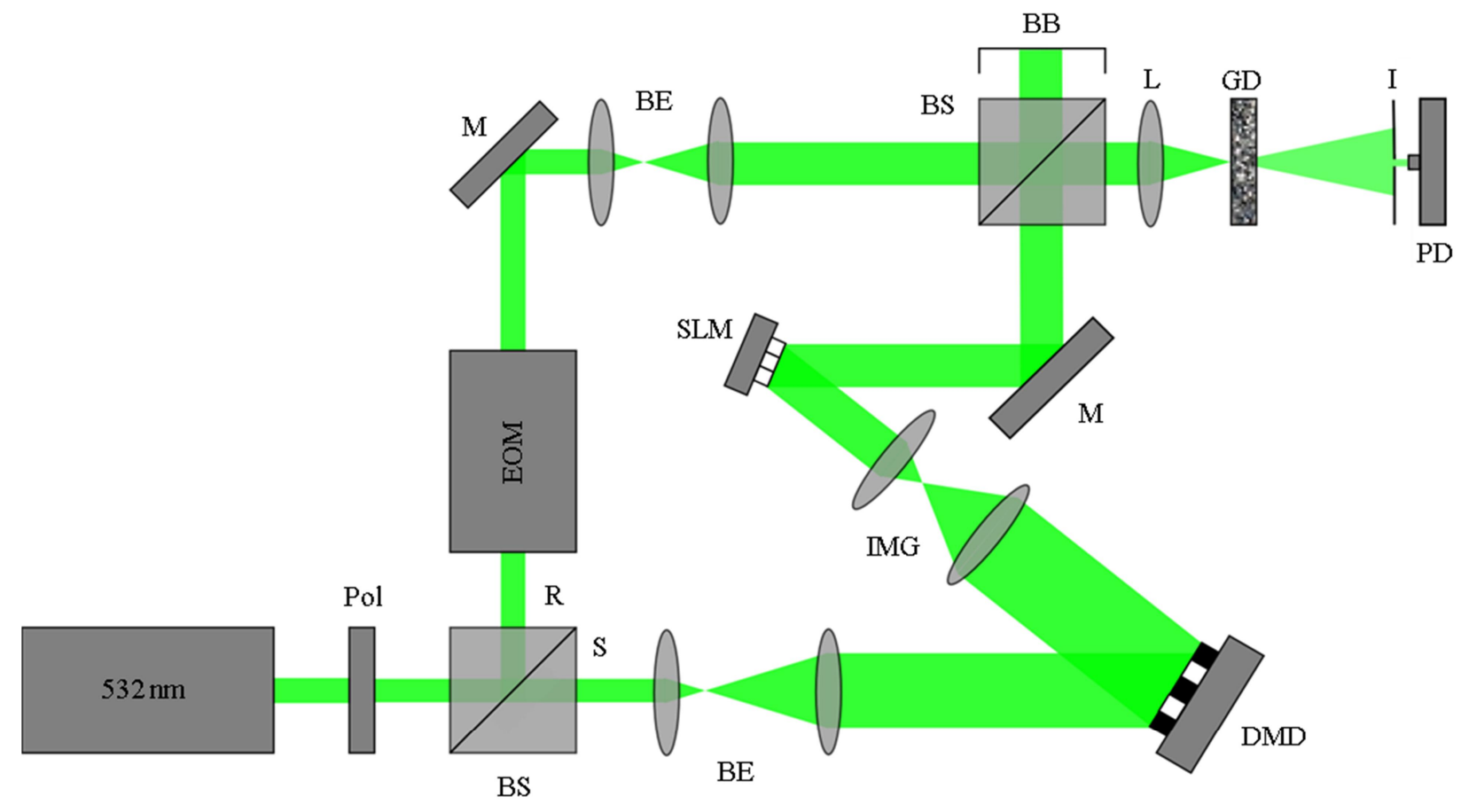

Fig. 1. Schematic of the optical system. BB, beam block; BE, beam expander; BS, beam splitter; DMD, digital micromirror; EOM, electro-optic modulator, GD, ground glass diffuser; I, iris; IMG, $4 \mathrm{f}$ imaging system; L, lens; M, mirror; PD, photodiode; Pol, polarizer; R, Reference arm; S, Sample arm; SLM, spatial light modulator.

The focusing procedure consisted of three steps: acquisition, calculation and display. During acquisition, only the DMD and function generator of the EOM were triggered, with the DAQ acquiring the signal from the photodiode at $10 \mathrm{MS} / \mathrm{s}$. A trigger was given by a separate output DAQ (NI 6321, National Instruments, USA) and cascaded by a second function generator (DG4162, Rigol Technologies, China) to produce a train of 65 TTL trigger pulses at $18.85 \mathrm{KHz}$ sent simultaneously to the EOM function generator, DMD and DAQ. Each trigger caused a new input mode to be illuminated in the manner of the step-wise algorithm ${ }^{30}$, while also rotating the phase of the reference beam and capturing the resultant signal at the photodiode. After all 64 input modes have been measured and the data transferred to the computer, the 65th trigger resulted in full illumination of the SLM by the DMD. The measurements were then processed via the CPU to determine the optimal phase for each input mode and the phase-map created and loaded to the SLM. Finally, another trigger was sent from the output DAQ to the second function generator and then passed to the DAQ and SLM. The optimized phase-map was then displayed and the resulting signal recorded by the DAQ.

\section{RESULTS}

Using our hybrid approach to collect measurements allows us to effectively perform phase-based wavefront shaping at the speed of the DMD. The LCoS SLM is updated only once in display, regardless of the number of input modes utilized, without sacrificing illumination as in off-axis holography. The primary objective in developing this approach was to achieve wavefront shaping at sufficient speed to allow for focusing in living tissue, without sacrificing gain and/or energy as in other methods. To do this, the system must therefore: 1) optimize the wavefront within the in vivo correlation time $(<10 \mathrm{~ms})$ and 2$)$ focus light with a gain greater than unity. In order to test the former, the ground glass diffuser was held stationary at the focus of the lens. The photodiode and iris were then positioned to isolate and detect a single speckle grain. Using 64 modes, we achieved a total runtime, i.e. measurement, processing, display and display capture, of $7.93 \pm 0.04 \mathrm{~ms}(\mathrm{n}=10)$, well below the $10 \mathrm{~ms}$ cutoff of our goal. Within this time, $20.78 \pm 1.5$ times improvement was seen; $41.3 \pm 3.0 \%$ of the theoretical maximum enhancement as given by Eq. $1 .{ }^{8}$ 


$$
\eta_{\max }=\frac{\pi}{4}\left(\frac{n_{i}}{n_{o}}-1\right)+1
$$

Where $n_{1}$ is the number of input modes and $\mathrm{n}_{\mathrm{o}}$ is the number of modes detected at the output. This is in good agreement with values obtained by previous studies with regard to the enhancement as a percentage of the theoretical maximum, and to our knowledge the fastest demonstration of iterative phase-based wavefront shaping to date.

\subsection{Decorrelating Media Experiment}

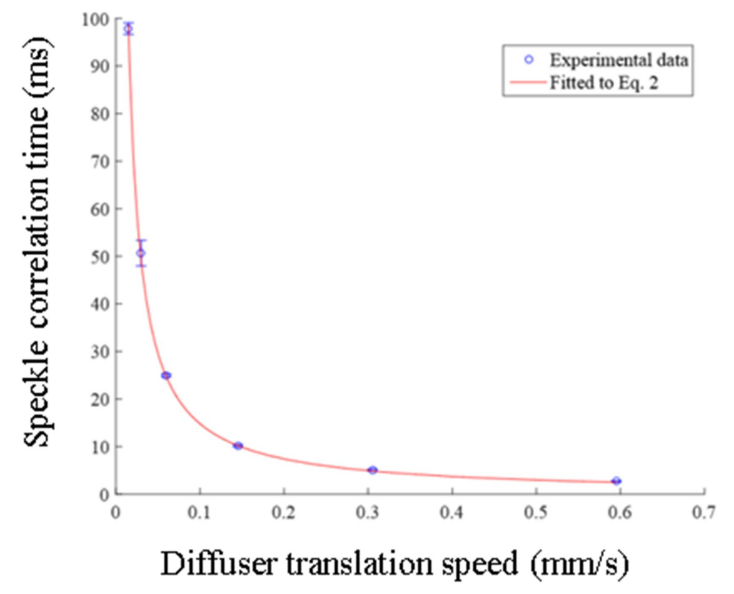

Fig. 2. Measurement and fit of the speckle correlation time at varying translations speeds. Error bars show the standard error of $\mathrm{T}_{\mathrm{c}}$ measurements $(\mathrm{n}=3)$.

The primary objective in developing this approach was to achieve wavefront shaping at sufficient speed to allow for focusing in living tissue. Here we demonstrate the capability of the hybrid system to focus through scattering media with varying correlation times, particularly those found within tissue in vivo. To produce replicable decorrelation, the ground glass diffuser was mounted on a variable velocity motorized translation stage (CONEX-LTA-HS, Newport, USA) as illustrated in Fig. 1. The line camera (Aviiva M2, Atmel, USA) then recorded the speckle field as the diffuser was translated at varying speeds. The correlation time, $T_{c}$, defined as the time for the correlation coefficient to reach $1 / \mathrm{e}^{2}$, was directly calculated for each speed by fitting the correlation coefficient of each frame with a Gaussian function ${ }^{28}$. The correlation time (ms) was then related to the translation speed, $v(\mathrm{~mm} / \mathrm{s})$, by the expected size of the speckle grain at the surface of the scattering media, $d_{b}=1.479 \mu \mathrm{m}$, as seen in Eq. 2 and illustrated in Fig. 2.

$$
T_{c}=\frac{d_{b}}{v}
$$

Speckle correlation times of 100, 50, 25, 10,5 and $2.5 \mathrm{~ms}$ were selected for testing, as well as a stationary control (Tc $>$ $10,000 \mathrm{~ms}$ ), and the corresponding speed of the stage determined from Eq. 2. The stage was then translated at the predetermined speed for each speckle correlation time as the hybrid system was used to shape the wavefront and generate a focus. Each individual measurement was performed at a different location on the diffuser, allowing for distinct paths of travel through the scattering medium and resulting speckle grain field at the plane of the photodiode. The control is seen to have a $13.91 \pm 0.84$ times enhancement, lower than the improvement previously discussed due to multiple measurements at various positions; some of which resulted in a non-optimal number of modes at the photodiode (i.e. $>$ 1). As demonstrated in Fig. 3, while improvement showed degradation as the correlation time decreased, improvement greater than unity was seen at all selected times. 


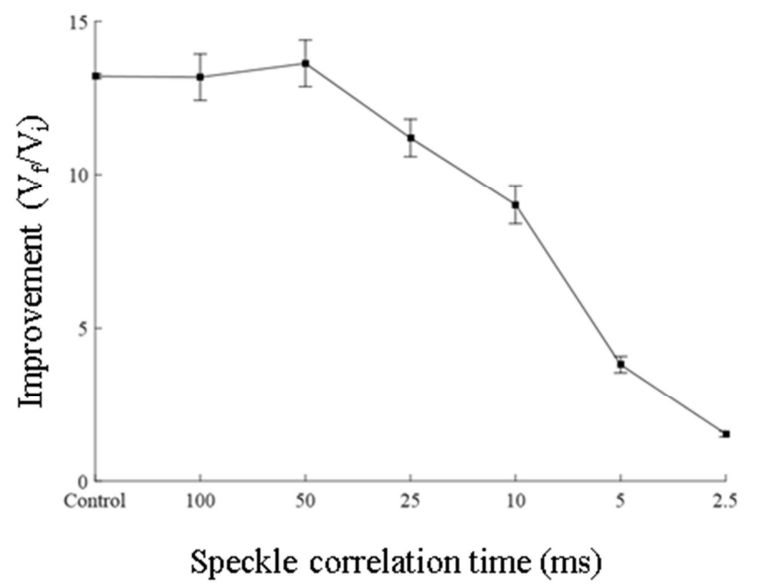

Fig. 3. Focusing through decorrelating a medium. Control represents the stationary diffuser with correlation time greater than $10,000 \mathrm{~ms}$. Error bars illustrate the standard error of improvement measurements $(\mathrm{n}=100)$.

\section{DISCUSSION}

Here, we have demonstrated a novel technique which hybridizes the wavefront shaping method, leveraging the respective advantages of the DMD and SLM while minimizing their disadvantages. We have illustrated the speed and efficacy of the hybrid system in two studies, demonstrating focusing in both stable and dynamic scattering media. First, focusing was tested through a stationary ground glass diffuser, achieving approximately $41 \%$ of the maximum theoretical enhancement in a runtime of under $8 \mathrm{~ms}$ using 64 superpixels. This is well within the range of in vivo correlation times, potentially providing the ability to focus through diffusive media with correlation times similar to living tissue. To verify, the ground glass diffuse was mounted on a variable speed motorized stage, allowing the correlation time of the scattering media to be manipulated. As previously shown, this study confirmed the capability of the system; providing improvement greater than unity at all tested correlation times.

The primary limitation of the hybrid system is currently the number of superpixels, which may make focusing in tissue challenging due to increased number of modes at the detector, which linearly decreases enhancement as expressed by Eq.1. However, because the wavefront shaping process is hybridized, additional input modes can be added at the speed of the DMD (about $0.055 \mathrm{~ms}$ per mode) with a fixed amount of time required for the SLM to load and update (approximately $4 \mathrm{~ms}$ ). The number of superpixels used is therefore a balance between the desired time of optimization and degrees of freedom, but may be freely changed as needed. As the number of input modes increase, the signal-tonoise ratio (SNR) may also become restrictive as each superpixel is isolated individually by the DMD and contributes illumination based upon the area of the SLM it covers. To correct this issue, it may become beneficial to implement a Hadamard basis as opposed to the simple modal isolation seen here. The Hadamard basis may provide additional SNR as well as higher gain by correcting for cross-talk between superpixels. Both the basis utilized and number of input modes may be changed in the current system with no further modifications needed to the optical layout.

In summary, the hybrid technique removes the need to compromise, in iterative wavefront shaping, to obtain the speed of the DMD. The use of the EOM in conjunction with the DMD permits phase-modulation in an on-axis optical layout. In turn, the high-speed of the system allows for runtimes demonstrated to allow focusing in dynamic media with correlation times similar to those found in living tissue. Combined with the elimination of the detrimental effects present in current high-speed techniques, the hybrid method is perhaps uniquely suited to in vivo wavefront optimization. While some issues must still be resolved, such as the number of input modes and further improvement of runtime; this potential promises to significantly advance biomedical optics, extending the depth of focusing through and, possibly, in biological tissue. 


\section{ACKNOWLEDGEMENTS}

This research was sponsored in part by National Institutes of Health grants DP1 EB016986 (NIH Director's Pioneer Award) and R01 CA186567 (NIH Director's Transformative Research Award). L. W. has a financial interest in Microphotoacoustics, Inc. and Endra, Inc. which, however, did not support this work.

\section{REFERENCES}

[1] Denk, W., Strickler, J. and Webb, W. "Two-photon laser scanning fluorescence microscopy," Science 248, 73-76 (1990).

[2] Pawley, J. [Handbook of Biological Confocal Microscopy], Springer, (2010).

[3] Huang, D., et al., "Optical coherence tomography," Science 254, 1178-1181 (1991).

[4] Fenno, L., Yizhar, O. and Deisseroth, K., "The development and application of optogenetics," Ann. Rev. Neurosci. 34, 389-412 (2011).

[5] Yanik, M. F., et al., "Neurosurgery: functional regeneration after laser axotomy," Nature 432, 822-822 (2004).

[6] Galanzha, E. I., et al., "In vivo fiber-based multicolor photoacoustic detection and photothermal purging of metastasis in sentinel lymph nodes targeted by nanoparticles," J. Biophoton. 2, 528-539 (2009).

[7] Wang, L. V., and Wu, H. I., [Biomedical optics: principles and imaging], John Wiley \& Sons, (2012).

[8] Vellekoop, I. M. and Mosk, A. P., "Focusing coherent light through opaque strongly scattering media," Opt. Lett. 32, 2309-2311 (2007).

[9] Conkey, D. B., Caravaca-Aguirre, A. M. and Piestun, R., "High-speed scattering medium characterization with application to focusing light through turbid media," Opt. Express 20, 1733-1740 (2012).

[10] Chaigne, T., Katz, O., Boccara, A. C., Fink, M., Bossy, E. and Gigan, S., "Controlling light in scattering media noninvasively using the photoacoustic transmission matrix," Nat. Photonics 8, 58-64 (2014).

[11] Tay, J. W., Lai, P., Suzuki, Y. and Wang, L. V., "Ultrasonically encoded wavefront shaping for focusing into random media," Sci. Rep. 4, 3918 (2014).

[12] Lai, P., Wang, L., Tay, J. W., and Wang, L.V., "Photoacoustically guided wavefront shaping for enhanced optical focusing in scattering media," Nat. Photonics 9, 126-132 (2015).

[13] Caravaca-Aguirre, A. M., Niv, E., Conkey, D. B. and Piestun, R., "Real-time resilient focusing through a bending multimode fiber," Opt. Express 21, 12881-12887 (2013).

[14] Akbulut, D., Huisman, T. J., van Putten, E. G., Vos, W. L. and Mosk, A. P., "Focusing light through random photonic media by binary amplitude modulation," Opt. Express 19, 4017-4029 (2011).

[15] Mosk, A. P., Lagendijk, A., Lerosey, G. and Fink, M., "Controlling waves in space and time for imaging and focusing in complex media," Nat. Photonics 6, 283-292 (2012).

[16] Hsieh, C. L., Pu, Y., Grange, R. and Psaltis, D., "Digital phase conjugation of second harmonic radiation emitted by nanoparticles in turbid media," Opt. Express 18, 12283-12290 (2010).

[17] Vellekoop, I. M. and Aegerter, C. M., "Scattered light fluorescence microscopy: imaging through turbid layers," Opt. Lett. 35, 1245-1247 (2010).

[18] Vellekoop, I. M., Cui, M. and Yang, C., "Digital optical phase conjugation of fluorescence in turbid tissue," Appl. Phys. Lett. 101, 081108 (2012).

[19] Xu, X., Liu, H. and Wang, L. V., "Time-reversed ultrasonically encoded optical focusing into scattering media," Nat. Photonics 5, 154-157 (2011).

[20] Si, K., Fiolka, R. and Cui, M., "Fluorescence imaging beyond the ballistic regime by ultrasound-pulse-guided digital phase conjugation," Nat. Photonics 6, 657-661 (2012).

[21] Wang, Y. M., Judkewitz, B., DiMarzio, C. A. and Yang, C., "Deep-tissue focal fluorescence imaging with digitally time-reversed ultrasound-encoded light," Nat. Commun. 3, 928 (2012).

[22] Angeliqu, A., Et al., "Reference-less measurement of the transmission matrix of a highly scattering material using a DMD and phase retrieval techniques." Optics express 23, 9 (2015)

[23] Lev, A. and Sfez, B., "In vivo demonstration of the ultrasound-modulated light technique." JOSA A 20, 12 (2003).

[24] Jang, M., et al., "Relation between speckle decorrelation and optical phase conjugation (OPC)-based turbidity suppression through dynamic scattering media: a study on in vivo mouse skin," Biomedical optics express 6, 1 (2015). 
[25] Zhou, E. H., Ruan, H., Yang, C. and Judkewitz, B., "Focusing on moving targets through scattering samples," Optica 1, 227-232 (2014).

[26] Yaqoob, Z., Psaltis, D., Feld, M. S. and Yang, C., "Optical phase conjugation for turbidity suppression in biological samples," Nat. Photonics 2, 110-115 (2008).

[27] Cui, M., McDowell, E. J. and Yang, C., "An in vivo study of turbidity suppression by optical phase conjugation (TSOPC) on rabbit ear," Opt. Express 18, 25-30 (2010).

[28] Liu, Y., Lai, P., Ma, C., Xu, X., Grabar, A. A., and Wang, L. V., "Optical focusing deep inside dynamic scattering media with near-infrared time-reversed ultrasonically encoded (TRUE) light," Nat. Commun. 6, 5904 (2015).

[29] Tay, J. W., Jinyang, L. and Wang, L. V., "Amplitude-masked photoacoustic wavefront shaping and application in flowmetry," Optics letters 39, 19 (2014).

[30] Vellekoop, I.M. and Mosk, A. P., "Phase control algorithms for focusing light through turbid media," Optics communications 281, 11 (2008). 